\title{
Do changes in illness perceptions, physical activity, and behavioural regulation influence fatigue severity and health-related outcomes in CFS patients?
}

\author{
V. De Gucht* F.K. Garcia, M. den Engelsman, S. Maes \\ Leiden University, Institute of Psychology, Health, Medical and Neuropsychology Unit, The Netherlands
}

\section{A R T I C L E I N F O}

\section{Article history:}

Received 21 June 2016

Received in revised form 15 February 2017

Accepted 16 February 2017

\section{Keywords:}

Chronic Fatigue Syndrome

Behavioural regulation patterns

Illness perceptions

Observational longitudinal study

\begin{abstract}
A B S T R A C T
Objective: Examine to what extent changes in cognitions and changes in physical activity and behavioural regulation patterns influence fatigue severity, physical symptoms, and physical and psychological functioning of patients suffering from Chronic Fatigue Syndrome (CFS) at follow-up.

Methods: The present study is an observational longitudinal study with a 12-month follow-up. A total of 144 CFS patients participated both at baseline and at follow-up. Four separate hierarchical regression analyses were conducted with fatigue, physical symptoms, physical functioning and psychological functioning at follow-up as the dependent variables, and (changes in) illness perceptions and behavioural regulation patterns (all-or-nothing and limiting behaviour) as the independent variables. Data were collected making use of self-report questionnaires.

Results: Increased Consequence and Identity beliefs over time, as well as increases in all-or-nothing behaviour predicted higher fatigue severity at follow-up. Both number and severity of physical symptoms and psychological functioning at follow-up were only determined by changes in illness perceptions, with increased Consequence beliefs influencing both outcomes, and increased Timeline beliefs only determining physical symptoms. Physical functioning at follow-up was predicted by changes in illness perceptions as well as increased levels of both all-ornothing and limiting behaviour.

Conclusion: The findings point at a differential pattern of associations between changes in illness perceptions and behaviour regulation patterns on the one hand, and patient outcomes on the other hand. Whereas illness perceptions significantly contribute to each of the outcomes, behaviour regulation patterns contribute only to fatigue severity and physical functioning.
\end{abstract}

(C) 2017 Elsevier Inc. All rights reserved.

\section{Introduction}

Chronic Fatigue Syndrome (CFS) is characterized by medically unexplained fatigue of at least six month duration, the fatigue has to be severe, disabling and lead to a significant reduction in level of occupational, personal and/or social functioning. CFS is diagnosed on the basis of the Oxford criteria [1] or the more restrictive Center for Disease Control (CDC) criteria [2]. According to the CDC criteria, the patient must also have reported at least four (out of eight) other somatic symptoms in addition to fatigue.

The prevalence of CFS ranges from $0.6 \%$ to $2.5 \%$, depending on the diagnostic criteria used, the setting (general population, primary, secondary, or tertiary care) and the country in which the studies were carried

\footnotetext{
* Corresponding author at: Leiden University, Institute of Psychology, Health, Medical and Neuropsychology Unit, Wassenaarseweg 52, 2333 AK Leiden, The Netherlands.

E-mail address: degucht@fsw.leidenuniv.nl (V. De Gucht).
}

out [3,4]. CFS seems to affect mainly young adults from 20 to 40 years old, the prevalence is two to three times higher in women, and seems to be comparable across socio-economic groups [4]. The prognosis of CFS is generally poor. In their review, Joyce and colleagues [5] state that $<10 \%$ of patients return to premorbid levels of functioning, a figure that was confirmed by a 3-year follow-up study [6]. While full recovery rates are low, there is a broader range in improvement rates among studies ranging from 6 to $63 \%$ [5].

Theoretical frameworks for CFS usually distinguish between factors that may render people more vulnerable to the development of CFS (predisposing factors), factors that may trigger CFS (precipitating factors) and factors that maintain CFS and impede recovery (perpetuating factors) $[7,8]$. The perpetuating factors are considered to be most important in view of the development of interventions as they may be responsible for the maintenance and, eventually, worsening of fatigue symptoms.

Existing models of perpetuating factors usually emphasize the role of psychological processes, especially cognitions and behaviours, in 
maintaining CFS $[9,10]$. These factors coincide with the key ingredients of the two treatment modalities that are considered to be efficacious for CFS according to the UK National Institute of Health and Clinical Excellence (NICE) guidelines on CFS [11] and to a number of meta-analyses on the subject [12-15]. These treatment modalities are: (a) Cognitive Behaviour Therapy (CBT), focusing on challenging dysfunctional cognitions related to fatigue/CFS, gradually increasing physical activity behaviour, and establishing a good activity/rest balance [16], and (b) Graded Exercise Training (GET) solely focusing on physical activity behaviour [13].

Recently, the question has been raised to what extent cognitions and behaviours mediate the effect of psychological and behavioural interventions (such as CBT and GET) on fatigue severity and patient functioning. On the basis of a re-analysis of three Randomized Controlled Trials (RCTs), examining the role of physical activity as a mediator of the effect of CBT on fatigue severity [17], it was demonstrated that changes in physical activity were not related to changes in fatigue. A similar result was found in a study by Heins et al. [18]. Another study [19] investigated whether the effect of CBT on fatigue and functional impairment was mediated by a cognitive factor (focusing on fatigue) and/or a behavioural factor (activity avoidance). A decreased tendency to focus on fatigue, but not a decreased tendency to avoid activity, was found to be a mediator of the effect of CBT on patient outcomes. Many studies therefore suggest that the efficacy of CBT for CFS is mediated by changes in cognitions and illness beliefs [17-20]. On the basis of these, but also earlier studies pointing out that cognitive dimensions were predictors of treatment effects [21,22], Knoop et al. [23] concluded that cognitions play a pivotal role in the maintenance of symptoms. More specifically, these authors' hypothesis is that an increase in physical activity, which is an important aspect of CBT, leads patients to change their perception of fatigue (with fatigue again becoming a normal sensation) and of the relation between fatigue and physical activity ("Despite my fatigue, I can be physically active."), which in turn has a positive influence upon fatigue-related outcomes. The results of a more recent study, looking into the mechanisms of change underlying CBT in CFS [24], seem to be in line with this hypothesis. More specifically, it was found that a path model where cognition acts as a mediator between behaviour and fatigue was statistically superior to a model where behaviour acts as a mediator between cognitions and fatigue.

The question is, however, whether the above mentioned hypothesis also applies to GET, where the focus of the intervention is solely on a gradual increase in level of physical activity. One study evaluating the effect of GET found that, within the intervention group, changes in illness perceptions, but not changes in fitness, were related to less fatigue and improved functioning after treatment [25]. Another study, evaluating a pragmatic rehabilitation intervention focusing upon gradual increases in level of physical activity, found however that both reductions in limiting behaviour and reductions in catastrophizing mediated treatment effects on fatigue [26].

Although the results of the above-mentioned intervention studies suggest that cognitive factors play a more central role than behavioural factors in predicting post-treatment fatigue and functioning in CFS patients, little is known about the role of cognitive and behavioural factors in the natural course of (long-standing) CFS. That is why we were interested in this study in examining if, and to what extent, changes in cognitions and behaviours over time predict changes in important patient outcomes. The research question of this study is therefore: "To what extent do changes in cognitions about fatigue (i.e. illness perceptions) and changes in behavioural factors (i.e. level of physical activity and behaviour regulation patterns) influence changes in fatigue severity, number and severity of physical symptoms, and (physical and emotional) health-related quality of life of CFS patients over a one-year follow-up period?"

\section{Methods}

\subsection{Design}

The present study is an observational longitudinal study with a 12 month follow-up. All patients participating in the study gave their written informed consent. Approval from the Ethical Committee of the Institute of Psychology at Leiden was obtained (20-1-2010/CEP255).

\subsection{Sample}

The participants for the study were recruited from a large Dutch patient organization. Initially an informational email was sent to 1800 members of the organization, containing information on the rationale and aim of the study. Three hundred and eighteen patients were interested in participating in the study; 261 patients (82\%) filled out the baseline questionnaires (T1). After baseline measurement, 35 patients (13.4\%) were excluded because they reported to have a chronic disease that could account for their fatigue and/or because they received psychological or psychiatric treatment for a severe psychiatric disorder. To check whether all patients in our sample fulfilled the CDC criteria for CFS [2], they filled out a CDC-based checklist for CFS [27]. As a result, 12 patients were excluded (4.5\%). The number of patients at T1 was thus reduced to 214 . At one-year follow-up, 144 patients (67\%) filled out the questionnaires a second time (T2). No significant differences were found between the patients that participated only at T1 and the patients that participated at both time points for age, gender, and illness duration.

\subsection{Measures}

\subsubsection{Independent variables}

Illness perceptions were measured using the validated Dutch version of the Brief Illness Perception Questionnaire (Brief IPQ-DLV) measuring (perceived) consequences, timeline, identity, personal control, treatment control, coherence, emotional representation, and cause [28]. Cause was not measured in this study. Emotional Representation is constructed by summing the responses, ranging from 1 to 10 , on two items, Concern and Mood. All other dimensions are measured with a single item and scored on a scale from 1 to 10 . For the subscales consequences, timeline, identity and emotional representation, higher scores represent more negative illness perceptions (e.g. more consequences or a longer timeline). For the subscales personal control, treatment control and coherence, higher scores represent more positive illness perceptions (e.g. more personal control or more treatment control). The Brief IPQ was shown to have good reliability and validity $[28,29]$. Both the original IPQ [30] and the brief IPQ discriminate well between patients suffering from different chronic conditions, including CFS [31].

Physical activity was determined with the Short Questionnaire to Assess Health-Enhancing Physical Activity (SQUASH) [32]. Patients were asked whether they engaged in physical activity such as walking, biking or sports and what kind of physical activity they performed. Patients were also asked to specify the frequency (days per week) and duration (hours and/or minutes per day) of that physical activity. For every specified physical activity the frequency (days) was multiplied by the duration (minutes). The total score for physical activity was calculated by summing the amount of minutes for all physical activities specified. Higher scores indicated more minutes of physical activity per week. The SQUASH has been found to be a reliable and valid questionnaire, and has been used in adults with chronic conditions, including CFS $[33,34]$.

Behaviour regulation patterns were assessed with the All-or-nothing and Limiting behaviour scales from The Behavioural Responses to Illness Questionnaire (BRIQ) [35]. The first dimension assesses the "boom and bust pattern" typically observed in CFS and the second dimension assesses the excessive rest that patients take due to their fatigue problems. 
Each item is scored on a scale from 1 to 5 . Higher scores represent more all-or-nothing behaviour and more limiting behaviour, respectively. The Dutch version of the BRIQ has a very good internal consistency for the All-or-Nothing behaviour subscale $(\alpha=0.88)$ and a lower, but acceptable one for the Limiting behaviour subscale ( $\alpha=0.72$ ) [36]. The BRIQ was designed as a predictive tool for medically unexplained symptoms and syndromes including CFS following acute infections [35].

\subsubsection{Dependent variables}

Fatigue Severity was measured with the Dutch version of the Checklist of Individual Strength (CIS-20R) [37,38]. The CIS-20R is a reliable and well-validated 20-item self-report questionnaire assessing four dimensions of fatigue: subjective experience of fatigue, lack of concentration, lack of motivation and activity reduction. The CIS-20 was developed for CFS patients and has good psychometric properties in this patient group $[37,38]$. Items are rated on a 7 -point Likert scale ranging from "Yes, that is true" to "No, that is not true". For the purpose of the present study the total fatigue severity score (ranging from 20 to 140) was used. The internal consistency of this total score is excellent $(\alpha=0.90)[37$, 38].

Severity of physical symptoms was measured by means of the Patient Health Questionnaire-15 (PHQ-15), assessing the presence and severity of 15 somatic symptoms [39]. A higher score indicates a higher level of somatization. The PHQ-15 is a valid and reliable measure of physical symptoms and is adequate to assess symptom severity in patients with medically unexplained symptoms and syndromes [40].

Health-related quality of life (QoL). The Short Form Health Survey-12 (SF-12 V.2) [41] was used to assess physical and psychological functioning. The SF-12v2 is a well validated measure consisting of 8 domains: general health perception, physical functioning, role limitations due to physical problems, bodily pain, vitality, role limitations due to emotional problems, social functioning and mental health. These 8 dimensions are combined into two sum scores, representing physical functioning (physical QoL) and psychological functioning (psychological QoL). For the purpose of the present study, only the sum scores for the two dimensions were used. Each sum score ranges from 0 to 100, with higher scores representing better QoL. The SF-12 has been used in many studies, also in patients with CFS. The SF-12 is seen as a standardized measure to assess QoL in CFS patients [42].

\subsubsection{Control variables}

The demographic variables, gender, age and illness duration were obtained from the baseline questionnaires.

\subsection{Data analysis}

Descriptive statistics and frequency analysis were performed to describe the study sample and the variables included in the study. Oneway repeated measures ANOVAs were conducted to investigate whether changes in illness perceptions and behavioural variables over time were statistically significant. In addition to $p$-values, Confidence Intervals were calculated. To examine whether changes in the independent variables were clinically relevant, effect sizes (Cohen's d) were calculated [43].

To answer the central research question, four separate hierarchical multiple regression analyses were conducted. Fatigue, physical symptoms, physical functioning and psychological functioning at follow-up (T2) were the dependent variables of the regression models. Within each of the regression models, demographic variables were entered as control variables in the first block of the model, followed by Illness duration in block two. To control for baseline levels of the outcome variable, the outcome variable at baseline (T1) was entered in the third block of the regression model. Changes $(\Delta)$ in illness perceptions and changes $(\Delta)$ in behaviour from baseline to follow-up (T2-T1) were entered into block four and block five of the regression model, respectively. Only the change variables that were significantly correlated with at least one outcome variable were entered into the regression models. Multicollinearity was assessed by examining the relationship between the independent variables in the Pearson's Correlation analysis and by calculating the Variance Inflation Factors (VIF) and Tolerance statistics. The analyses were conducted using SPSS version 21 for windows.

\section{Results}

Only participants who met the CDC criteria at $\mathrm{T} 1$ and who completed the questionnaires at both time points were included in the study $(N=$ 144). For all variables included in the study, with the exception of physical activity, $<10 \%$ of the data was missing $(1.3 \%-8.3 \%)$. For these variables, no missing data techniques were used [44]. For the variable physical activity, $13.2 \%$ of the data was missing. For this variable, missing values were imputed by means of Mean Imputation.

\subsection{Sample characteristics}

The demographic characteristics of the sample are shown in Table 1. Eighty-four percent of the patients were female. The average age was 44.65 ( $S D=11.89$ ). Forty-eight percent of the patients completed higher education. Two thirds of patients were currently not working. Patients reported an average Illness duration of 13.49 years (ranging from 1.29 to 40 years).

\subsection{Descriptive statistics and univariate analysis}

The descriptive statistics and the results of the one-way repeatedmeasures ANOVA are displayed in Table 2.

The results of the one-way repeated measures ANOVA showed that the illness perceptions, consequences, identity, coherence and emotional representation changed significantly over the one-year follow-up period. These changes were however not clinically relevant [43]. Of the behavioural variables, only limiting behaviour changed significantly over time. This change was clinically relevant, but small [43].

Table 3 shows the results of the correlation analysis between the change scores of the variables included in the study. The change scores for (1) consequences, (2) timeline, (3) personal control, (4) identity, (5) coherence, (6) emotional representation, (7) all-or-nothing behaviour, and (8) limiting behaviour, were significantly correlated with the change scores of at least one outcome variable. These change scores $(\Delta)$ were entered as independent variables into the hierarchical multiple regression analysis.

No multicollinearity was found between the change scores, included as independent variables in the hierarchical regression analyses (Table 3 ). The results of the correlation analysis showed small to large correlation coefficients [45], with the highest correlation being between $\Delta$ identity and $\Delta$ consequences $(r=0.56)$. The results of the VIF and Tolerance statistics are reported below.

Table 1

Demographic characteristics at baseline $(N=144)$.

\begin{tabular}{lll}
\hline & $N$ & $\%$ \\
\hline Gender & & \\
$\quad$ Female & 121 & 84 \\
$\quad$ Male & 23 & 16 \\
Age $(M, S D)$ & $44.64(11.89)$ & \\
Educational level & & \\
$\quad$ Primary education & 23 & 16 \\
$\quad$ Secondary education & 52 & 36.1 \\
$\quad$ Higher education & 69 & 47.9 \\
Working status & & 32.6 \\
$\quad$ Working & 47 & 67.4 \\
$\quad$ Not working & 97 & \\
Illness duration, years $(M, S D)$ & $13.49(8.27)$ & \\
$\quad$ Number of additional somatic symptoms $(M, S D)$ & $6.18(1.27)$ & \\
$\quad$ & &
\end{tabular}


Table 2

Descriptive statistics and changes in illness perceptions and behavioural variables from baseline (T1) to follow-up (T2).

\begin{tabular}{|c|c|c|c|c|c|c|}
\hline & $\mathrm{T} 1$ & $\mathrm{~T} 2$ & $95 \% \mathrm{CI}$ of difference & $F$ & $p$ & $d$ \\
\hline \multicolumn{7}{|l|}{ Illness perceptions } \\
\hline Consequence & $8.97(1.33)$ & $8.53(1.71)$ & $0.18-0.70$ & 11.16 & 0.001 & 0.073 \\
\hline Timeline & $8.23(1.75)$ & $8.47(1.72)$ & $-0.49-0.01$ & 3.38 & $\overline{0.068}$ & 0.024 \\
\hline Personal control & $4.95(2.07)$ & $5.08(2.09)$ & $-0.46-0.19$ & 0.064 & 0.420 & 0.005 \\
\hline Treatment control & $4.74(2.38)$ & $4.74(2.37)$ & $-0.43-0.42$ & 0.00 & 0.974 & 0.000 \\
\hline Identity & $8.16(1.33)$ & $7.81(1.58)$ & $0.12-0.58$ & 9.18 & 0.003 & 0.061 \\
\hline Coherence & $6.56(2.34)$ & $5.98(2.44)$ & $0.12-1.03$ & 6.22 & $\overline{0.014}$ & 0.042 \\
\hline Emotional representation & $11.94(4.14)$ & $11.32(3.85)$ & $0.05-1.21$ & 4.55 & $\overline{0.035}$ & 0.031 \\
\hline \multicolumn{7}{|l|}{ Behavioural variables } \\
\hline Physical activity & $216.16(231.09)$ & $175.70(175.72)$ & $-2.86-83.78$ & 3.42 & 0.067 & 0.165 \\
\hline All-or-nothing behaviour & $13.53(4.34)$ & $13.04(4.55)$ & $-0.22-1.19$ & 1.88 & 0.172 & 0.117 \\
\hline Limiting behaviour & $20.15(4.21)$ & $18.75(4.44)$ & $0.71-2.10$ & 15.85 & 0.000 & 0.340 \\
\hline \multicolumn{7}{|l|}{ Dependent variables } \\
\hline Fatigue & $101.18(16.31)$ & $97.54(17.04)$ & & & & \\
\hline Physical symptoms & $13.45(4.09)$ & $12.79(4.32)$ & & & & \\
\hline Physical functioning & $36.16(13.53)$ & $37.85(15.65)$ & & & & \\
\hline Psychological functioning & $51.87(14.47)$ & $53.56(15.86)$ & & & & \\
\hline
\end{tabular}

\subsection{Hierarchical regression analyses}

The results of the hierarchical multiple regression analyses for fatigue, physical symptoms, functional status, and psychological functioning are displayed in Table 4. All results are reported while controlling for demographic variables (block 1) and illness duration (block 2). For all four regression analyses, the VIF and tolerance ranged from 1.00 to 1.57 and 0.64 to 1.00 , respectively.

\subsubsection{Changes in fatigue}

Female gender was significantly associated with a decrease in fatigue from baseline to follow-up. Fatigue at baseline explained $24.7 \%$ of the variance in fatigue at $\mathrm{T} 2$. Changes in illness perceptions explained an additional $14.7 \%$ of the variance in fatigue at $\mathrm{T} 2$. Increased consequence and identity beliefs over time were significantly and independently associated with higher levels of fatigue at T2. Changes in behavioural variables added 3.5\% to the explained variance in fatigue at T2. Increased scores for All-or-Nothing behaviour over time were associated with higher levels of fatigue at $\mathrm{T} 2$. The total variance explained by the model was $53.9 \%$.

\subsubsection{Changes in physical symptoms}

Baseline levels of physical symptoms were significantly associated with physical symptoms at T2. Physical symptoms at baseline explained $51.8 \%$ of the variance in physical symptoms at $\mathrm{T} 2$. Changes in illness perceptions explained an additional $9.2 \%$ of the variance in physical symptoms at T2. Increased consequence and timeline beliefs over time were associated with higher physical symptoms at T2. Changes in behavioural variables were not significantly associated with physical symptoms at T2. The total variance explained by the model was $63.0 \%$.

\subsubsection{Changes in physical functioning}

Physical functioning at baseline explained $44.4 \%$ of the variance in physical functioning at T2. Changes in illness perceptions as a whole explained an additional $6.8 \%$ of the variance in physical functioning at T2. The individual predictors did, however, not explain enough variance in the outcome variable, physical functioning, to be statistically significant. Changes in behavioural variables added $4.6 \%$ to the explanation of the variance in Physical Functioning at T2. Increased scores for both Allor-Nothing behaviour and Limiting Behaviour over time were associated with lower physical functioning at T2. The total variance explained by the model was $58.2 \%$.

\subsubsection{Changes in psychological functioning}

Psychological functioning at baseline explained $31.4 \%$ of the variance in psychological functioning at T2. Changes in illness perceptions explained an additional $8.3 \%$ of the variance in psychological functioning at T2. Increased consequence beliefs over time were associated with lower psychological functioning at T2. Changes in behavioural variables were not significantly associated with psychological functioning at T2 $(p>0.05)$. The total variance explained by the model was $42.7 \%$.

Table 3

Correlations between change variables.

\begin{tabular}{|c|c|c|c|c|c|c|c|c|c|c|c|c|c|c|}
\hline & 1 & 2 & 3 & 4 & 5 & 6 & 7 & 8 & 9 & 10 & 11 & 12 & 13 & 14 \\
\hline 1. $\Delta$ consequence & - & & & & & & & & & & & & & \\
\hline 2. $\Delta$ timeline & $0.22^{* *}$ & - & & & & & & & & & & & & \\
\hline 3. $\Delta$ personal control & $-0.24^{* *}$ & 0.01 & - & & & & & & & & & & & \\
\hline 4. $\Delta$ treatment control & 0.09 & -0.09 & -0.01 & - & & & & & & & & & & \\
\hline 5. $\Delta$ identity & $0.56^{* *}$ & 0.12 & $-0.24^{* *}$ & 0.03 & - & & & & & & & & & \\
\hline 6. $\Delta$ coherence & -0.12 & -0.01 & $0.27^{* *}$ & -0.05 & $-0.19^{*}$ & - & & & & & & & & \\
\hline 7. $\Delta$ emotional representation & $0.23^{* *}$ & 0.12 & $-0.25^{* *}$ & 0.04 & $0.32^{* *}$ & $-0.17^{*}$ & - & & & & & & & \\
\hline 8. $\Delta$ minutes of physical activity & -0.03 & $0.21^{*}$ & 0.06 & -0.03 & -0.01 & -0.08 & 0.07 & - & & & & & & \\
\hline 9. $\Delta$ all-or-nothing behaviour & $0.19^{*}$ & 0.09 & -0.04 & 0.02 & 0.06 & 0.06 & 0.16 & -0.01 & - & & & & & \\
\hline 10. $\Delta$ limiting behaviour & $0.23^{* *}$ & 0.04 & $-0.19^{*}$ & -0.06 & 0.16 & -0.04 & 0.09 & -0.09 & -0.04 & - & & & & \\
\hline 11. $\Delta$ fatigue & $0.41^{* *}$ & 0.03 & $-0.22^{* *}$ & -0.06 & $0.39^{* *}$ & -0.10 & $0.17^{*}$ & -0.03 & $0.25^{* *}$ & $0.29^{* *}$ & - & & & \\
\hline 12. $\Delta$ physical symptoms & $0.27^{* *}$ & $0.22^{*}$ & -0.12 & -0.02 & $0.29^{* *}$ & 0.01 & 0.13 & -0.11 & $0.19^{*}$ & 0.14 & $0.25^{* *}$ & - & & \\
\hline 13. $\Delta$ physical functioning & $-0.34^{* *}$ & -0.10 & $0.25^{* *}$ & 0.10 & $-0.34^{* *}$ & 0.12 & $-0.25^{* *}$ & 0.10 & $-0.25^{* *}$ & $-0.28^{* *}$ & $-0.36^{* *}$ & $-0.20^{* *}$ & - & \\
\hline 14. $\Delta$ psychological functioning & $-0.33^{* *}$ & -0.14 & $0.21^{* *}$ & 0.07 & $-0.19^{*}$ & $0.21^{*}$ & $-0.34^{* *}$ & 0.07 & -0.16 & $-0.17^{*}$ & $-0.28^{* *}$ & $-0.23^{* *}$ & $0.40^{* *}$ & - \\
\hline
\end{tabular}

\footnotetext{
* $p<0.05$
}

** $p<0.01$. 
Table 4

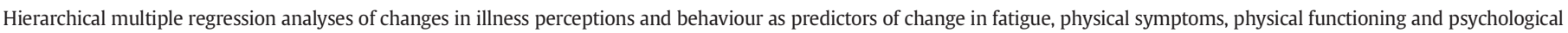
functioning.

\begin{tabular}{|c|c|c|c|c|c|c|c|c|}
\hline & \multicolumn{2}{|l|}{ Fatigue at $\mathrm{T} 2$} & \multicolumn{2}{|c|}{ Physical symptoms at T2 } & \multicolumn{2}{|c|}{ Physical functioning at T2 } & \multicolumn{2}{|c|}{$\begin{array}{l}\text { Psychological functioning at } \\
\text { T2 }\end{array}$} \\
\hline & $\Delta \mathrm{R}^{2}$ & $\beta$ & $\Delta \mathrm{R}^{2}$ & $\beta$ & $\Delta \mathrm{R}^{2}$ & $\beta$ & $\Delta \mathrm{R}^{2}$ & $\beta$ \\
\hline Block 1 (demographic variables) & $0.092^{* *}$ & & 0.004 & & 0.013 & & 0.008 & \\
\hline Gender & & $-0.15^{*}$ & & -0.07 & & 0.03 & & 0.13 \\
\hline Age & & 0.09 & & 0.06 & & $-0.15^{*}$ & & 0.03 \\
\hline Block 2 (clinical characteristics) & 0.018 & & 0.000 & & 0.013 & & 0.017 & \\
\hline Illness duration at $\mathrm{T} 1$ & & -0.09 & & -0.05 & & 0.14 & & -0.07 \\
\hline Block 3 (dependent at T1) & $0.247^{* * *}$ & $0.58^{* * *}$ & $0.518^{* * *}$ & $0.68^{* * *}$ & $0.444^{* * *}$ & $0.68^{* * *}$ & $0.314^{* * *}$ & $0.62^{* * * *}$ \\
\hline Block 4 (illness perceptions) & $0.147^{* * *}$ & & $0.092^{* *}$ & & $0.068^{*}$ & & $0.083^{*}$ & \\
\hline$\Delta$ consequence & & $0.20^{*}$ & & $0.15^{*}$ & & -0.09 & & $-0.18^{*}$ \\
\hline$\Delta$ timeline & & -0.07 & & $0.14^{*}$ & & -0.00 & & -0.04 \\
\hline$\Delta$ personal control & & -0.09 & & -0.03 & & 0.10 & & 0.05 \\
\hline$\Delta$ identity & & $0.24^{* *}$ & & 0.11 & & -0.07 & & 0.06 \\
\hline$\Delta$ coherence & & 0.07 & & 0.09 & & 0.00 & & 0.08 \\
\hline$\Delta$ emotional representation & & -0.09 & & -0.06 & & -0.05 & & -0.15 \\
\hline Block 5 (behaviour) & $0.035^{*}$ & & 0.015 & & $0.046^{* *}$ & & 0.006 & \\
\hline$\Delta$ all-or-nothing behaviour & & $0.18^{* *}$ & & 0.12 & & $-0.14^{*}$ & & -0.08 \\
\hline$\Delta$ limiting behaviour & & 0.08 & & 0.06 & & $-0.18^{* *}$ & & 0.01 \\
\hline $\mathrm{R}^{2}(\operatorname{adj})$ & $0.539(0.489)$ & & $0.630(0.588)$ & & $0.582(0.537)$ & & $0.427(0.365)$ & \\
\hline
\end{tabular}

$\Delta$, change scores between $\mathrm{T} 1$ and $\mathrm{T} 2$.

* $p<0.05$.

** $p<0.01$.

*** $p<0.001$

\section{Discussion}

Over a one-year follow-up period, an increase in fatigue severity is mainly influenced by changes in illness perceptions (an increase in perceived consequences as well as in the number of symptoms attributed to CFS), and, to a lesser degree, by an increase in the boom-and-bust behavioural pattern. In addition, females perceived significantly less fatigue at follow-up. Changes in number and severity of physical symptoms over the same time period are solely determined by changes in illness perceptions: higher perceived consequences and a longer expected duration of CFS independently contribute to an increase in physical symptoms over time. Both physical and psychological functioning at follow-up is influenced by changes in illness perceptions over time. None of the changes in separate illness perceptions however contribute significantly and independently to physical functioning, whereas worse psychological functioning at follow-up is determined by an increase in perceived consequences. In addition, both an increase in all-or-nothing behaviour and limiting behaviour independently contribute to worse physical functioning over time. The duration of CFS at baseline (ranging from 1.29 to 40 years) is not related to any of the outcomes at follow-up. Likewise, changes in level of physical activity over time are not significantly associated with any of the outcomes at follow-up.

The above findings clearly point at a differential pattern of associations between changes in cognitive factors (i.e. illness perceptions) and behaviour regulation patterns on the one hand, and changes in outcomes on the other hand. Changes in illness perceptions significantly contribute to each of the outcomes, but the variance explained by these changes is higher for fatigue severity at T2 than for the other outcomes. With the exception of physical functioning, perceiving more adverse consequences over time contributes to all outcomes. In addition, attributing more symptoms to CFS predicts more severe fatigue and expecting a longer illness duration predicts more severe physical symptoms at follow-up. Each of these illness perceptions seems to be related to perceived illness severity. The underlying concept could be a negative or pessimistic view of the illness, which has a detrimental effect on the course of the illness. As changes in illness perceptions are too small to be clinically relevant these findings should however be interpreted with caution.

Changes in behaviour regulation patterns appear to contribute only to fatigue severity and to physical functioning. An increase in the boom-and-bust pattern contributes to both higher fatigue severity and worse physical functioning, whereas an increase in limiting or resting behaviour only contributes to worse physical functioning at follow-up.

The fact that changes in illness perceptions significantly contribute to each of the outcomes is in accordance with previous studies [18,25]. The finding that changes in behavioural factors contribute to some, but not all outcomes, is, however, not in accordance with the results of earlier studies, with the exception of the pragmatic rehabilitation study [26], as all other studies demonstrated that changes in behavioural factors did not mediate treatment effects on fatigue [18,19,24]. This may be explained by differences in both study design and measurement of fatigue. The existing studies that have examined the role of cognitive and behavioural factors as active ingredients of change were intervention studies aiming at improving fatigue through changing cognitions and activity patterns, whereas our study is a prospective cohort study, that looks into the natural evolution of symptoms and functioning over time. In addition, in the present study, the total fatigue severity score was used, taking into account the different dimensions that, as a whole, make up the fatigue concept, whereas other studies only included subjective fatigue [18,19], or self-rated improvement of fatigue (YES/ NO) as an outcome [25]. Measuring only subjective fatigue is however not congruent with the common point of view that fatigue is a multidimensional concept which includes a physical and a mental dimension $[4,38,46,47]$ as well as a dimension that refers to lack of motivation to start any activity $[4,38,47]$. A fatigue severity score should therefore include all these fatigue dimensions. Using only one dimension, or using a binary fatigue recovery item as an outcome may, for obvious reasons, lead to different results.

Changes in level of physical activity are not related to any of the outcomes. This finding is in accordance with previous studies, showing that physical activity was not a mediator of treatment effect on fatigue severity in CFS $[17,18,48]$. The fact that changes in behaviour regulation patterns significantly contributed to both fatigue severity and physical functioning at follow-up, points to the fact that dysfunctional behavioural patterns are a better predictor of fatigue severity and impaired functioning than physical activity because they more adequately assess (im)balance between activity and rest, which is typically related to physical deconditioning.

With respect to illness perceptions, especially changes in perceived consequences, identity, and timeline beliefs significantly contribute to 
the outcomes. Most of the studies that looked into the association between illness perceptions and relevant patient outcomes in CFS were cross-sectional. The two dimensions most consistently found to be related to fatigue (or lack of vitality), physical functioning and psychological well-being (or distress) were perceiving more severe consequences of CFS and attributing a lot of somatic symptoms to CFS [49-51] which is in accordance with the results of the present study. The few existing prospective studies were conducted in samples consisting of patients who were diagnosed with infectious mononucleosis and focused on predicting if and to what extent illness perceptions were capable of predicting who developed $\mathrm{CF}(\mathrm{S})$ over time $[52,53]$. To the best of our knowledge, there are no studies that investigated the contribution of changes in illness perceptions to progression of fatigue severity and worsening of physical and psychological functioning in patients with (long-standing) CFS.

The same accounts for the relationship between maladaptive behaviour regulation patterns and patient outcomes in CFS. 'All-or-nothing behaviour', measuring a boom-and-bust pattern of activity, was found to be the most significant predictor of the development of CFS 6 months after an acute episode of glandular fever [53]. In addition, 'limiting behaviour', measured post-treatment, mediated the positive effects of a pragmatic rehabilitation intervention on fatigue at one-year follow-up in CFS patients [26]. Although both studies point to the potential importance of maladaptive behaviour regulation patterns in CFS, there are no observational longitudinal studies that examined their significance for fatigue-related outcomes in patients who have already been diagnosed with CFS.

\subsection{Strengths and limitations of the study}

The major strength of this study is the fact that it is a prospective study in a group of CFS patients that is large enough at follow-up to test our central hypothesis. Moreover, this is the first study in CFS patients that has investigated whether 'natural' changes in illness perceptions, physical activity, and behavioural regulation patterns over a oneyear follow-up period are related to relevant patient outcomes. Despite this, several remarks can be made with respect to this study.

The follow-up period is only one year. This is an important limitation of the study. A longer systematic follow-up period of e.g. five years would (a) most probably show a greater amount of change in both the predictors and the outcomes over time and (b) allow for a repeated measures design whereby patients are followed systematically over time. While we found statistically significant changes over time for some illness perceptions, none of them are clinically significant. With respect to the behavioural factors only limiting behaviour showed a clinically relevant change. A longer follow-up period could remediate this limitation.

Another important limitation is that this study is conducted in a CFS population reporting a mean disease duration of over 13 years. Changes in such a population probably require more time and effort than changes in a patient group that was recently diagnosed with CFS. In addition, the determinants of change may differ between patients with a longer and a shorter illness duration as suggested by the results of Brown et al. [54]. Independent of this, a systematic follow-up study starting as early as possible after the diagnosis of CFS/ME would be optimal to identify predictors that can be the target of early interventions as interventions (as e.g. CBT and GET) are probably more effective in changing perceptions and behaviour in earlier than in later stages of the syndrome.

In addition, the present study was conducted in patients that were recruited from a large patient support organization. Previous studies suggest that these patients may differ from other CFS patients, since they usually report a higher frequency of CFS-related symptoms [55] and more psychological distress [56] than persons with CFS in the general population. This may limit the generalizability of the results.
Finally, in the present study we only examined the contribution of changes in some cognitive and behavioural factors to patient outcomes. Future studies should not only extend the number and type of cognitive (e.g. catastrophizing or self-efficacy) and behavioural factors (e.g. sleep) that are included, but should also investigate the role of emotional and social factors, as they could also contribute to changes in patient outcomes over time [57].

\section{Competing interest statement}

The authors have no competing interests.

\section{Acknowledgements}

We would like to thank the members of the patient organization (ME/CVS Stichting Nederland) for their participation in the study.

\section{References}

[1] M.C. Sharpe, L.C. Archard, J.E. Banatvala, L.K. Borysiewicz, A.W. Clare, A. David, et al., A report-chronic fatigue syndrome: guidelines for research, J. R. Soc. Med. 84 (1991) 118-121.

[2] K. Fukuda, S.E. Straus, I. Hickie, M.C. Sharpe, J.G. Dobbins, A. Komaroff, The chronic fatigue syndrome: a comprehensive approach to its definition and study. International Chronic Fatigue Syndrome Study Group, Ann. Intern. Med. 121 (1994) 953-959, http://dx.doi.org/10.7326/0003-4819-121-12-199412150-00009.

[3] M. van't Leven, G.A. Zielhuis, J.W. van der Meer, A.L. Verbeek, G. Bleijenberg, Fatigue and chronic fatigue syndrome-like complaints in the general population, Eur. J. Pub. Health 20 (2010) 251-257, http://dx.doi.org/10.1093/eurpub/ckp113.

[4] A. Avellaneda Fernandez, A. Perez Martin, M. Izquierdo Martinez, M. Arruti Bustillo F.J. Barbado Hernandez, Labrado J. de la Cruz, et al., Chronic fatigue syndrome: aetiology, diagnosis and treatment, BMC Psychiatry 9 (Suppl. 1) (2009) S1, http:// dx.doi.org/10.1186/1471-244x-9-s1-s1.

[5] J. Joyce, M. Hotopf, S. Wessely, The prognosis of chronic fatigue and chronic fatigue syndrome: a systematic review, QJM 90 (1997) 223-233, http://dx.doi.org/10.1093/ qjmed/90.3.223.

[6] R. Nisenbaum, J.F. Jones, E.R. Unger, M. Reyes, W.C. Reeves, A population-based study of the clinical course of chronic fatigue syndrome, Health Qual. Life Outcomes 1 (2003) 49, http://dx.doi.org/10.1186/1477-7525-1-49.

[7] S.B. Harvey, S. Wessely, Chronic fatigue syndrome: identifying zebras amongst the horses, BMC Med. 7 (2009) 58, http://dx.doi.org/10.1186/1741-7015-7-58.

[8] B. Van Houdenhove, P. Luyten, Customizing treatment of chronic fatigue syndrome and fibromyalgia: the role of perpetuating factors, Psychosomatics 49 (2008) 470-477, http://dx.doi.org/10.1176/appi.psy.49.6.470.

[9] J.H. Vercoulen, C.M. Swanink, J.M. Galama, J.F. Fennis, P.J. Jongen, O.R. Hommes, et al., The persistence of fatigue in chronic fatigue syndrome and multiple sclerosis: development of a model, J. Psychosom. Res. 45 (1998) 507-517, http://dx.doi.org/10. 1016/s0022-3999(98)00023-3.

[10] A.M. Fry, M. Martin, Fatigue in the chronic fatigue syndrome: a cognitive phenomenon? J. Psychosom. Res. 41 (1996) 415-426, http://dx.doi.org/10.1016/s00223999(96)00190-0.

[11] National Institute for Health and Clinical Excellence, Chronic Fatigue Syndrome/myalgic Encephalomyelitis (Or Encephalopathy): Diagnosis and Management of CFS/ ME in Adults and Children, NICE, London, 2007 (http://guidance.nice.org.uk/ CG053).

[12] J.R. Price, E. Mitchell, E. Tidy, V. Hunot, Cognitive behaviour therapy for chronic fatigue syndrome in adults, Cochrane Database Syst. Rev. (2008), CD001027. http:// dx.doi.org/10.1002/14651858.cd001027.pub2.

[13] L. Larun, K.G. Brurberg, J. Odgaard-Jensen, J.R. Price, Exercise therapy for chronic fatigue syndrome, Cochrane Database Syst. Rev. 2 (2016), CD003200. http://dx.doi. org/10.1002/14651858.cd003200.pub4.

[14] B.D. Castell, N. Kazantzis, R.E. Moss-Morris, Cognitive behavioral therapy and graded exercise for chronic fatigue syndrome: a meta-analysis, Clin. Psychol. Sci. Pract. 18 (2011) 311-324, http://dx.doi.org/10.1111/j.1468-2850.2011.01262.x.

[15] M.M. Marques, V. De Gucht, M.J. Gouveia, I. Leal, S. Maes, Differential effects of behavioral interventions with a graded physical activity component in patients suffering from chronic fatigue (syndrome): an updated systematic review and metaanalysis, Clin. Psychol. Rev. 40 (2015) 123-137, http://dx.doi.org/10.1016/j.cpr. 2015.05.009.

[16] G. Bleijenberg, J. Prins, E. Bazelmans, Cognitive behavioral therapies, in: L.A. Jaons, P.A. Fennel, R.R. Taylor (Eds.), Handbook of Chronic Fatigue Syndrome, Wiley, Hoboken, New Jersey 2003, pp. 493-526.

[17] J.F. Wiborg, H. Knoop, M. Stulemeijer, J.B. Prins, G. Bleijenberg, How does cognitive behaviour therapy reduce fatigue in patients with chronic fatigue syndrome? The role of physical activity, Psychol. Med. 40 (2010) 1281-1287, http://dx.doi.org/10. 1017/s0033291709992212.

[18] M.J. Heins, H. Knoop, W.J. Burk, G. Bleijenberg, The process of cognitive behaviour therapy for chronic fatigue syndrome: which changes in perpetuating cognitions and behaviour are related to a reduction in fatigue? J. Psychosom. Res. 75 (2013) 235-241, http://dx.doi.org/10.1016/j.jpsychores.2013.06.034. 
[19] J.F. Wiborg, H. Knoop, J.B. Prins, G. Bleijenberg, Does a decrease in avoidance behavior and focusing on fatigue mediate the effect of cognitive behavior therapy for chronic fatigue syndrome? J. Psychosom. Res. 70 (2011) 306-310, http://dx.doi. org/10.1016/j.jpsychores.2010.12.011.

[20] J.F. Wiborg, H. Knoop, L.E. Frank, G. Bleijenberg, Towards an evidence-based treatment model for cognitive behavioral interventions focusing on chronic fatigue syndrome, J. Psychosom. Res. 72 (2012) 399-404, http://dx.doi.org/10.1016/j. jpsychores.2012.01.018.

[21] A. Deale, T. Chalder, S. Wessely, Illness beliefs and treatment outcome in chronic fatigue syndrome, J. Psychosom. Res. 45 (1998) 77-83, http://dx.doi.org/10.1016/ s0022-3999(98)00021-X.

[22] J.B. Prins, G. Bleijenberg, E. Bazelmans, L.D. Elving, T.M. de Boo, J.L. Severens, et al., Cognitive behaviour therapy for chronic fatigue syndrome: a multicentre randomised controlled trial, Lancet 357 (2001) 841-847, http://dx.doi.org/10 1016/s0140-6736(00)04198-2.

[23] H. Knoop, J.B. Prins, R. Moss-Morris, G. Bleijenberg, The central role of cognitive processes in the perpetuation of chronic fatigue syndrome, J. Psychosom. Res. 68 (2010) 489-494, http://dx.doi.org/10.1016/j.jpsychores.2010.01.022.

[24] D. Stahl, K.A. Rimes, T. Chalder, Mechanisms of change underlying the efficacy of cognitive behaviour therapy for chronic fatigue syndrome in a specialist clinic: a mediation analysis, Psychol. Med. 44 (2014) 1331-1344, http://dx.doi.org/10. 1017/s0033291713002006.

[25] R. Moss-Morris, C. Sharon, R. Tobin, J.C. Baldi, A randomized controlled graded exercise trial for chronic fatigue syndrome: outcomes and mechanisms of change, J. Health Psychol. 10 (2005) 245-259, http://dx.doi.org/10.1177/1359105305049774.

[26] A.J. Wearden, R. Emsley, Mediators of the effects on fatigue of pragmatic rehabilitation for chronic fatigue syndrome, J. Consult. Clin. Psychol. 81 (2013) 831-838, http://dx.doi.org/10.1037/a0033561.

27] D. Wagner, R. Nisenbaum, C. Heim, J.F. Jones, E.R. Unger, W.C. Reeves, Psychometric properties of the CDC Symptom Inventory for assessment of chronic fatigue syndrome, Popul. Health Metrics 3 (2005) 8, http://dx.doi.org/10.1186/1478-7954-3-8.

[28] E.J. de Raaij, C. Schroder, F.J. Maissan, J.J. Pool, H. Wittink, Cross-cultural adaptation and measurement properties of the Brief Illness Perception Questionnaire-Dutch Language Version, Man. Ther. 17 (2012) 330-335, http://dx.doi.org/10.1016/j. math.2012.03.001.

[29] E. Broadbent, K.J. Petrie, J. Main, J. Weinman, The brief illness perception questionnaire, J. Psychosom. Res. 60 (2006) 631-637, http://dx.doi.org/10.1016/j. jpsychores.2005.10.020.

[30] J. Weinman, K. Petrie, R. Moss-Morris, R. Horne, The Illness Perception Questionnaire: a new method for assessing the cognitive representation of disease, Psychol. Health 11 (1996) 431-445, http://dx.doi.org/10.1080/08870449608400270.

[31] E. Broadbent, C. Wilkes, H. Koschwanez, J. Weinman, S. Norton, K.J. Petrie, A systematic review and meta-analysis of the Brief Illness Perception Questionnaire, Psychol. Health (2015) 1-74, http://dx.doi.org/10.1080/08870446.2015.1070851.

[32] G.C. Wendel-Vos, A.J. Schuit, W.H. Saris, D. Kromhout, Reproducibility and relative validity of the short questionnaire to assess health-enhancing physical activity, J. Clin. Epidemiol. 56 (2003) 1163-1169.

[33] M. Marques, V. De Gucht, I. Leal, S. Maes, Effects of a self-regulation based physical activity program (the "4-STEPS") for unexplained chronic fatigue: a randomized controlled trial, Int. J. Behav. Med. 22 (2015) 187-196, http://dx.doi.org/10.1007/ s12529-014-9432-4.

[34] N. Ungar, J. Wiskemann, M. Sieverding, Physical activity enjoyment and self-efficacy as predictors of cancer patients' physical activity level, Front. Psychol. 7 (2016) 898 http://dx.doi.org/10.3389/fpsyg.2016.00898.

[35] M. Spence, R. Moss-Morris, T. Chalder, The Behavioural Responses to Illness Questionnaire (BRIQ): a new predictive measure of medically unexplained symptoms following acute infection, Psychol. Med. 35 (2005) 583-593, http://dx.doi.org/10. 1017/s0033291704003484.

[36] M. Marques, V. De Gucht, I. Leal, S. Maes, A cross-cultural perspective on psychological determinants of chronic fatigue syndrome: a comparison between a Portuguese and a Dutch patient sample, Int. J. Behav. Med. 20 (2013) 229-238, http://dx.doi. org/10.1007/s12529-012-9265-y.

[37] J. Vercoulen, M. Alberts, G. Bleijenberg, De checklist individual strength (CIS), Gedragstherapie 32 (1999) 31-36.
[38] J.H. Vercoulen, C.M. Swanink, J.F. Fennis, J.M. Galama, J.W. van der Meer, G. Bleijenberg, Dimensional assessment of chronic fatigue syndrome, J. Psychosom. Res. 38 (1994) 383-392, http://dx.doi.org/10.1016/0022-3999(94)90099-x.

[39] K. Kroenke, R.L. Spitzer, J.B. Williams, The PHQ-15: validity of a new measure for evaluating the severity of somatic symptoms, Psychosom. Med. 64 (2002) 258-266, http://dx.doi.org/10.1097/00006842-200203000-00008.

[40] K. Kroenke, R.L. Spitzer, J.B. Williams, B. Löwe, The patient health questionnaire somatic, anxiety, and depressive symptom scales: a systematic review, Gen. Hosp. Psychiatry 32 (2010) 345-359, http://dx.doi.org/10.1016/j.genhosppsych.2010.03.006.

[41] J.E. Ware, M. Kosinski, D.M. Turner-Bowker, B. Gandek, How to Score Version 2 of the SF-12® Health Survey, Lincoln, RI, Quality Metric Incorporated, 2002.

[42] M. Smith, H. Nelson, E. Haney, M. Pappas, M. Daeges, N. Wasson, et al., Diagnosis and Treatment of Myalgic Encephalomyelitis/chronic Fatigue SyndromeEvidence Report/Technology Assessment No. 219. (Prepared by the Pacific Northwest Evidence-based Practice Center under contract 290-2012-00014-I. AHRQ Publication No. 15- E001-EF.) Agency for Healthcare Research and Quality, Rockville, MD, December 2014 (Accessed at https://www.ncbi.nlm.nih.gov/books/NBK293931/pdf/ Bookshelf_NBK293931.pdf on 12 February 2017).

[43] B. Middel, R. Stewart, J. Bouma, E. van Sonderen, W.J. van den Heuvel, How to validate clinically important change in health-related functional status. Is the magnitude of the effect size consistently related to magnitude of change as indicated by a global question rating? J. Eval. Clin. Pract. 7 (2001) 399-410.

[44] D.A. Bennett, How can I deal with missing data in my study? Aust. N. Z. J. Public Health 25 (2001) 464-469, http://dx.doi.org/10.1111/j.1467-842x.2001.tb00294.x.

[45] J. Cohen, Statistical Power Analysis for the Behavioral Sciences, second ed. Erlbaum, Hillsdale, NJ, 1988.

[46] T. Chalder, G. Berelowitz, T. Pawlikowska, L. Watts, S. Wessely, D. Wright, et al., Development of a fatigue scale, J. Psychosom. Res. 37 (1993) 147-153, http://dx.doi. org/10.1016/0022-3999(93)90081-p.

[47] E.M. Smets, B. Garssen, B. Bonke, J.C. De Haes, The Multidimensional Fatigue Inventory (MFI) psychometric qualities of an instrument to assess fatigue, J. Psychosom. Res. 39 (1995) 315-325, http://dx.doi.org/10.1016/0022-3999(94)00125-0.

[48] F. Friedberg, S. Sohl, Cognitive-behavior therapy in chronic fatigue syndrome: is improvement related to increased physical activity? J. Clin. Psychol. 65 (2009) 423-442, http://dx.doi.org/10.1002/jclp.20551.

[49] R. Moss-Morris, K.J. Petrie, J. Weinman, Functioning in chronic fatigue syndrome: Do illness perceptions play a regulatory role? Br. J. Health Psychol. 1 (1996) 15-25, http://dx.doi.org/10.1111/j.2044-8287.1996.tb00488.x.

[50] M.J. Heijmans, Coping and adaptive outcome in chronic fatigue syndrome: importance of illness cognitions, J. Psychosom. Res. 45 (1998) 39-51, http://dx.doi.org/ 10.1016/s0022-3999(97)00265-1.

[51] R. Edwards, R. Suresh, S. Lynch, P. Clarkson, P. Stanley, Illness perceptions and mood in chronic fatigue syndrome, J. Psychosom. Res. 50 (2001) 65-68, http://dx.doi.org/ 10.1016/s0022-3999(00)00204-X.

[52] B. Candy, T. Chalder, A.J. Cleare, A. Peakman, A. Skowera, S. Wessely, et al., Predictors of fatigue following the onset of infectious mononucleosis, Psychol. Med. 33 (2003) 847-855, http://dx.doi.org/10.1017/s0033291703007554.

[53] R. Moss-Morris, M.J. Spence, R. Hou, The pathway from glandular fever to chronic fatigue syndrome: can the cognitive behavioural model provide the map? Psychol. Med. 41 (2011) 1099-1107, http://dx.doi.org/10.1017/s003329171000139x.

[54] M.M. Brown, A.A. Brown, L.A. Jason, Illness duration and coping style in chronic fatigue syndrome, Psychol. Rep. 106 (2010) 383-393, http://dx.doi.org/10.2466/pr0. 106.2.383-393.

[55] L.A. Jason, A.V. Plioplys, S. Torres-Harding, K. Corradi, Comparing symptoms of chronic fatigue syndrome in a community-based versus tertiary care sample, J. Health Psychol. 8 (2003) 459-464, http://dx.doi.org/10.1177/13591053030084005.

[56] W.J. Katon, E. A. Walker, The relationship of chronic fatigue to psychiatric illness in community, primary care and tertiary care samples, Ciba Found. Symp. 173 (2007) 193-211, http://dx.doi.org/10.1002/9780470514382.ch12 (discussion - 11).

[57] S. Hempel, D. Chambers, A.M. Bagnall, C. Forbes, Risk factors for chronic fatigue syndrome/myalgic encephalomyelitis: a systematic scoping review of multiple predictor studies, Psychol. Med. 38 (2008) 915-926, http://dx.doi.org/10.1017/ S0033291707001602. 